\title{
A Short-Living Video: Self-Presentation Via the Instagram Story and its Implications
}

\author{
Kaiyang Guo ${ }^{1}$ \\ ${ }^{I}$ School of Information Engineering, Crean Lutheran High School, Irvine, California, America
}

\begin{abstract}
The current study aimed to investigate how posting stories on Instagram influence users' mental health and extrapolate the relationship between ephemeral social media use and users' psychological needs. Several Instagram stories were selected and analyzed in terms of content, emotion and attention-degree. The study discovered that people are likely to share Instagram stories to meet their goal of impression management, gather social support by connecting with their social network and eliciting feedback, which in turn can facilitate emotional well-being.
\end{abstract}

Keywords: Instagram Story, Ephemeral media, Emotional Well-being

\section{INTRODUCTION}

With the prevalence of communication technologies, social media has penetrated every aspect of human lives. On a daily basis, people use social media to share their emotions and activities within their social networks [6]. Often, these posts are persistent and recordable, indicting the prolonged existence of social information across space and time[4][17]. This feature of persistency has an impact on the process of information production, which is related to users' perception of self-presentational concerns [3][18]. However, the temporal features of social media have varied across platforms and platforms. For instance, the posts on one's Facebook timeline are of prolonged accessibility to people's social networks unless profile owners actively deleted it. In contrast, Snapchat allows users to share one moment with their friends which will be disposed of right after the receiver opens it. Previous scholarship identified this feature as ephemerality, referring to the design of erasing information posts after a short period[1].

Growing social media applications adopted this feature and gained popularity among users. These include Snapchar, Instagram story, and newly developed Facebook story. Bayer[1] and his colleagues suggested that sharing shortmoments on Snapchat was associated with positive mood and had a positive influence on users' emotions and relationship satisfaction, indicating the relationship ephemerality and affective process. However, these days the Instagram story has gained larger and larger popularity, with 500 million active users posting their stories daily with Instagram users spending 30 minutes daily on this platform[2]. It is of theoretical importance to note that Instagram story is essentially different from Snapchat. On one hand, it is accessible to others as long as 24 hours, which is somewhat longer than the accessibility of Snapchat. On the other hand, the Instagram story reached a wide array of audiences. Usually, the Instagram story is not only accessible to one's social network, but also the public depending on the profile owner's privacy settings. The paper intends to review how these distinctive features influence users' posting behaviors.

Besides, there is a growing interest in the implications of social media use on emotional well-being. A 2017 study indicates that Instagram is the most detrimental social network site on mental health among young adults[16]. However, it only revealed a correlation between the time of using a certain platform and mental health outcomes without elaborating on the underlying mechanisms. The purpose of this study is to explore the impact of story publishing on Instagram on users' mental health, especially the emotional process, whether negative or positive.

\section{SELF-PRESENTATION AND MEDIA AFFORDANCES}

Self-presentation refers to the act of conveying preferred information or desired image about self to the audience and being often tailored to various contexts[10]. To leave a desirable impression, people need to constantly monitor their communication behaviors with others. These required efforts pertain to self-presentation management[5]. One of the major goals during daily social interactions is whether the self-presented information will maintain consistently over time. In a large public setting, people often want to communicate an ideal self to align with the group norms or attain positive feedback from the audience. However, information delivered within a large social network can hold the presenter accountable and these public claims sometimes even lead to interpersonal repercussions. For example, when one wants to present himself/herself as optimistic filled with positive emotions in public, it is normally expected that he or she will maintain this trait throughout the course. Yet, it is inevitable to experience negative effects. How to exert such emotions and share with others become a self-presentation burden, because that is countering against their prior self-presented images. To breach these publicized traits, people might risk their longheld reputation and experience serious interpersonal repercussions. Since then, people with this continuous 
concern have to pay extensive attention to their selfpresentation.

With the prevalence of communication technologies today, self-presentation management often takes place online as well. Certain features of social media play critical roles in the process of self-presentation. As one of the major features, recordability often increases self-presentation concerns. People are afraid that their recorded statements or pictures will put them in a difficult situation. Different from traditional face-to-face interaction, social media will put everything on record. If these statements or pictures become controversial or countering one's self-image, social media profile owners will suffer from interpersonal risks or breaches of self-presentations. Moreover, what scares people the most is that these recordable content could be found even after they deleted them. Thus, people have to be extra cautious, when it comes to share or post any information on recordable social media.

Since recordable social media becomes an issue to people's self-presentation concerns, many social media companies come up with the solution by adding an ephemeral feature to their applications, such as Snapchat, Instagram story, and so on. Ephemeral refers to access to the information that only remains for a short time. For example, the Instagram story will stay for 24 hours after posting. The two major advantages for self-presentation of an ephemeral medium are non-recordable and disposable. Different from recordable social media platforms, ephemeral social media allows users to share their thoughts, emotions, and daily activities without main concerns over self-presentation. Because it is relatively hard to record these public statements and/or later hold presenters accountable for this information. Previous research points out that posting on Snapchat and similar ephemeral social media are associated with positive mood and enjoyable affection[1]. However, it is unclear what emotions or psychological needs are associated with the behavior of posting on ephemeral social media like the Instagram story.

Nowadays, social media users intend to gain psychological needs in their medium. In light of the aforementioned selfpresentation concerns, the most common need is protecting their self-presentation and self-image in front of their followers or friends on social media. In this case, people tend to take advantage of media features for their selfpresentation management, so it is of research and practical interest to explore how ephemeral medium can meet users' particular needs. Therefore, this paper aims to draw upon the previous framework in this regard and extrapolate the relationship between ephemeral social media use and users' psychological needs.

\section{THE IMPLICATIONS OF MEDIA AFFORDANCES}

How the environment provides or offers the possibility for certain human actions are known as affordances across various disciplines[9]. For communication studies, media affordances often refer to the action possibilities that the inherent functional features of medium provide[8][12]. Taking the different affordances between texting and $\mathrm{FtF}$ as an example, the message is either written (e.g., texting) or transmitted acoustically (e.g., FtF), or only textual-based information is available (e.g., texting) or both verbal and non-verbal cues are used [19]. In light of arguments regarding self-presentation concerns and media use, only those media affordances that users are aware of are important to fulfill their goals for impression management. Thus, this study defined media affordances as perceived media attributes and features that are useful in the user's media selection to attain their goals and needs[6].

As previously reviewed[1], people take the media affordance of ephemeral (or recordability) into consideration and engage in different activities of impression management across various media platforms. However, most of the extant research did not incorporate the perceived media affordances with their users' psychological needs for impression management. One research on media selection for sharing emotional events might be the most relevant to the present study. This study recruited undergraduate students to take part in daily diary surveys for 7 consecutive days and asked them to report the most significant positive or negative event that day and identify which media they used to discuss these incidents[6]. Analyses showed that the high bandwidth channel, like $\mathrm{FtF}$, was more preferred for sharing intense negative events, as the availability of nonverbal cues could be effective in providing instant comfort, support or reassurance from trusted ones. Since then the study drawing inferences between the media use patterns and psychological needs. That is, since people utilize media in the way described above, they must be consciously considering the importance of these affordances. In an attempt to further advance this line of research, this study intends to select several stories and explore ephemerality afforded by the Instagram story and examine its relationship with users' needs for impression management in terms of content, emotion and attention-degree and its further implication on users' affective process.

\section{IMPRESSION MANAGEMENT AND EPHEMERAL MEDIUM}

Ephemeral medium is frequently used for sports fans. Their prediction or trash talk will not be recorded so there's no repercussion to them. Some studies found that the ephemeral medium is particularly useful for the selfpresentational purpose among sports fun[19]. Users are more likely to share negative experiences and their team identification for a short period of time. This is because people would not like to protect their own "faces", especially when it comes to express their support for their teams and/or players. To spread negative information against a rivalry team/player or publicly endorse their own team/player can be embedded with certain risks, such as personal repercussions or inciting quarrels fights with others. To avoid such risks, the affordance of ephemerality 
becomes handy since the posts are not recordable to a certain extent. Free from such constraints, sports fans might be more willing to share negative information and team identification through the ephemeral medium.

However, an unaddressed research gap is whether people will implement such self-presentational strategies in general, when they share their daily activities, emotionalcharged events or simply exchange information with their friends. By drawing inferences from previous literature, the paper proposed that people will strategically utilize the ephemeral medium to share negative and/or negative information to elicit social support, which in turn helps them cope with such emotions and further lead to increasing implementation of such self-presentational strategies for their Instagram stories.

\section{SELF-PRESENTATIONAL CONCERNS AND IMPRESSION MANAGEMENT}

As the study discovered, the Instagram story with its ephemeral affordance can meet users' needs for selfpresentational concerns and impression management. It is likely that people strategically utilize Instagram story to share emotional events to achieve the goal of ego protection. Traditionally, studies show that people tend to share their positive emotions on non-intrusive media, for example, texting or Facebook. When it comes to sharing negative feelings, phone calling or $\mathrm{FtF}$ is usually a more favored option. The point is that these two ways could help people to vent out their negative emotions and receive emotional support. However, the Instagram story could fulfill this need. Posting thoughts as stories can be viewed by many people. The people who have the same opinion will show support to the person who posts.

Extant literature on the association between sharing negative events and emotional well-being are inconsistent. Sharing emotions appears to alleviate the emotional upset experienced by the negative event, and thus contributes to emotional relief and psychological well-being. A vast body of research has investigated the emotional repercussions that arise from the sharing of negative emotional events as a coping process. For example, participants assigned to talk about their negative emotions reported more subjective benefits than control participants [21].

Sharing negative emotions also involves interpersonal processes, as one talks about something to someone. Therefore, the validation and support offered by partners of social sharing may lead to the alleviation of negative emotions[13]. Indeed, sharing negative emotion was associated with positive relationship outcomes, including receiving support and heightening of intimacy in the relationships[11]. These positive effects of social sharing are known to be a gradual long-term process rather than an immediate benefit as it takes time to process it and recover from the event[14]. The emotional repercussions of sharing negative events are dependent upon time. These findings suggest that sharing negative events can produce psychological benefits in the long run, as the sharer processes the event.

Because of the self-presentation concerns mentioned earlier, people are more likely to select Instagram stories as their platform to share their negative emotions due to its ephemeral feature. People often face events that are upsetting, so they will need an outlet for these negative feelings. How could an Instagram story with its ephemeral features meet these needs to share negative emotions without concerning their self-presentation? Previous research has documented certain findings shedding light on this question. For example, Bayer and his colleagues[1]found out that by posting Snapchat and ephemeral posts, people are later associated with the positive affective experience. Other scholars suggested that image-based social media posts could create positive effects on mental health, including positive emotions, less loneliness and less depressive symptoms[15]. So it is plausible that this is since people can receive social support and positive feedback from posting such events on social media. It is plausible to stipulate posting on the Instagram story can have similar effects. So this paper proposed that by sharing these events on the Instagram story, people can receive social support and feedback.

\section{ELICIT FEEDBACK AND GATHER SOCIAL SUPPORT}

Additionally, another advantage of sharing emotional events via Instagram stories can reach out to a large audience instantaneously. In this way, people who share their negative emotions on the Instagram story can gather social support in a short period of time by making connections with their network and eliciting feedback from their resources.

Scholarship on emotional well-being and social media users have long shown that social support is an important construct for social media users to facilitate their emotional well-being. Social support refers to the resources individual gathered from their interpersonal relationships, such as family members, significant others, friends and so on. It is social media today that largely connects individuals with their social network and provides convenient access to elicit social support. Certainly, the general sense of connection and belongingness has a beneficial effect on well-being. However, a more delicate mechanism, termed as the buffering hypothesis of self-disclosure, indicates that people who share or express their concerns and thoughts are more likely to extract the benefits of social support. It is easy to hypothesize that those who share positive emotions are likely to contagiously spread positivity and reaffirm their affective reactions through social media via social support. However, negative disclosure can be tricky due to the individual's self-presentation concerns and the availability of social support.

When people are dealing with negative emotions, they have the innate need to elicit social support and interpersonal resources from their existing social network. To extract 
social support, individuals can enjoy some beneficial outcomes for their emotional well-being. For instance, when one's flight was delayed, the person was trapped in the airport for a long time without knowing how much longer the waiting remained. People have a variety of ways to cope with such stressful situations filled with negative emotions. There was a group of people who constantly talk with the counter agent next to the gate to keep updated with information about the delay. This informational support can be soothing and reassuring which helps individuals regulate this anxious feeling.

\section{EMOTIONAL REGULATION AND POSITIVE EMOTIONAL OUTCOMES}

Some other groups of people may choose to do it differently. They would like to use media to reach out to their significant social network to elicit emotional support. This type of support from their network can be instantly helpful to alleviate stress when facing such emergency and difficult situations. In general, social support plays a significant buffering role when people under emotional events and situations, accounting for good emotional regulation and emotional well-being. As previously noted, the prevalence of social media affords people to utilize these platforms to easily reap the benefits of social support with convenient accessibility and instantaneous connection.

It has been long proven that social support leads to better emotional well-being[7][16]. Overall, this feedback and support will potentially serve as resources for emotional regulation and affection adjustment, which can lead to more positive emotional outcomes.

\section{CONCLUSION}

Based on previous arguments and propositions, this paper argues that the need for self-presentation leads to users' perceived higher value of ephemeral affordance of Instagram stories. Thus, people are likely to share positive and negative emotional events through Instagram stories to meet their goal of impression management. Moreover, using such a medium to share emotional events is useful for individuals to gather social support by connecting with their social network and eliciting feedback, which in turn can facilitate emotional well-being. Overall, the paper illuminates the optimistic side of social media use, by highlighting the connection between psychological needs and technological affordances.

\section{REFERENCES}

[1] Bayer, J. B., Ellison, N. B., Schoenebeck, S. Y., \& Falk, E. B. (2016). Sharing the small moments: ephemeral social interaction on Snapchat. Information, Communication \& Society, 19(7), 956-977.
[2] Bell, K. (2017). We're more addicted to Instagram than ever and it's all because of Stories. Retrieved from https://mashable.com/2017/08/02/instagram-stories-oneyear/?europe $=$ true $\# 8 \mathrm{vrfBx} 1 \mathrm{mMaq} 6$

[3] Berger, J. (2013). Beyond viral: Interpersonal communication in the internet age. Psychological Inquiry, 24(4), 293-296.

[4] Boyd, d. (2011). Social network sites as networked publics: Affordances, dynamics, and implications. In Z. Papacharissi (Ed.), A networked self (pp. 39-58). New York: Routledge.

[5] Brown, P., \& Levinson, S. C. (1987). Politeness: Some universals in language usage (Rev. ed.). Cambridge, UK: Cambridge University Press.

[6] Choi, M., \& Toma, C. L. (2014). Social sharing through interpersonal media: Patterns and effects on emotional wellbeing. Computers in Human Behavior, 36, 530-541.

[7] Cohen, S., \& Wills, T. A. (1985). Stress, social support, and the buffering hypothesis. Psychological Bulletin, 98(2), 310.

[8] Fox, J., \& McEwan, B. (2017). Distinguishing technologies for social interaction: The perceived social affordances of communication channels scale. Communication Monographs, 84, 298-318.

[9] Gibson, J. J. (1977). The concept of affordances. In R. Shaw \& J. Bransford (Eds.), Perceiving, acting, and knowing (pp. 67-82). Hillsdale, NJ: Lawrence Erlbaum.

[10] Goffman, E. (1967). Interaction ritual: Essay on faceto-face behavior. New York: Pantheon.

[11] Graham, S. M., Huang, J. Y., Clark, M. S., \& Helgeson, V. S. (2008). The positives of negative emotions: Willingness to express negative emotions promotes relationships. Personality and Social Psychology Bulletin, 34(3), 394-406.

[12] Kraut, R. E., Fussell, S. R., Brennan, S. E., \& Siegel, J. (2002). Understanding effects of proximity on collaboration: Implications for technologies to support remote collaborative work. Distributed work, 137-162.

[13] Nils, F., \& Rimé, B. (2012). Beyond the myth of venting: Social sharing modes determine the benefits of emotional disclosure. European Journal of Social Psychology, 42(6), 672-681.

[14] Pennebaker, J. W., \& Seagal, J. D. (1999). Forming a story: The health benefits of narrative. Journal of clinical psychology, 55(10), 1243-1254. 
[15] Pittman, M., \& Reich, B. (2016). Social media and loneliness: Why an Instagram picture may be worth more than a thousand Twitter words. Computers in Human Behavior, 62, 155-167.

[16] Ross, C. E. (2017). Social causes of psychological distress. Abingdon, United Kingdom: Routledge.

[17] Treem, J. W., \& Leonardi, P. (2012). Social media use in organizations: Exploring the affordances of visibility, editability, persistence, and association. Communication Yearbook, 36, 143-189.

[18] Vitak, J. (2012). The impact of context collapse and privacy on social network site disclosures. Journal of Broadcasting \& Electronic Media, 56(4), 451-470.

[19] Wakefield, L. T., \& Bennett, G. (2018). Sports fan experience: Electronic word-of-mouth in ephemeral social media. Sport Management Review, 21(2), 147-159.

[20] Walther, J. B. (1996). Computer-mediated communication: Impersonal, interpersonal, and hyperpersonal interaction. Communication research, 23(1), 3-43.

[21] Zech, E., \& Rimé, B. (2005). Is talking about an emotional experience helpful? Effects on emotional recovery and perceived benefits. Clinical Psychology \& Psychotherapy: An International Journal of Theory \& Practice, 12(4), 270-287. 